\title{
Kinetic Alfvén mode and kinetic magnetosonic mode from a fluid description
}

\author{
Glenn J. Beach" and Y. Y. Lau \\ Department of Nuclear Engineering, University of Michigan, Ann Arbor, Michigan 48109-2104
}

(Received 13 September 1994; accepted 18 January 1995)

\begin{abstract}
The dispersion relations for the classical electromagnetic modes in a uniform, magnetized, monoenergetic plasma, are reconstructed from a fluid approach. Under study are the Alfvén waves (parallel propagation) and the magnetosonic waves (perpendicular propagation). This fluid theory accounts for finite Larmor radius effects to all order, and is shown to yield identical results from the Vlasov formulation. (c) 1995 American Institute of Physics.
\end{abstract}

\section{INTRODUCTION}

In a previous paper, ${ }^{1}$ hereafter called I, the classical electrostatic modes, such as the Bernstein modes, the loss cone modes, and the Harris dispersion relation are reconstructed from a fluid theory. The theory begins with the assumption of a monoenergetic plasma. By simply calculating the displacement $\mathbf{x}_{1}$ of a fluid element from its equilibrium position $\mathbf{x}_{0}$, the charge perturbation density $n_{1}$, and the dispersion relation, immediately follow. The effect of a general equilibrium distribution function is included only at the end, by a trivial superposition of the result obtained for the monoenergetic plasma. Finite Larmor radius effects to all order and kinetic effects are fully accounted for, as this fluid theory is shown to yield identical results from the solution to the linearized Vlasov equation.

In this paper, we extend the fluid theory to treat electromagnetic modes, where the magnetic field perturbations are important. We need to calculate the perturbation current density $\mathbf{J}_{1}$, in response to a small signal electromagnetic field. It suffices to demonstrate the equivalence between the fluid and the Vlasov approach for a monoenergetic plasma, as the result for a general equilibrium distribution function may be obtained from a trivial superposition, as in I. As is well known, and explicitly demonstrated here once more, the dispersion relation is simply a statement of the conservation of energy-the electromagnetic energy of the mode is derived from the work done by the fluid displacement $x_{1}$ on the small signal electromagnetic field. This dispersion relation will be derived for both parallel propagating waves (Alfvén mode) and perpendicular propagating waves (magnetosonic mode). For both cases, it is shown to be identical to the Vlasov formulation.

In Sec. II, we describe the model. In Secs. III and IV, we use the fluid theory to derive the dispersion relations, respectively, for the kinetic Alfvén waves and for the kinetic magnetosonic waves. The corresponding Vlasov theories are given in the Appendices. Discussions of some interesting issues are given in Sec. V.

a)Present address: FAAC Inc., 825 Victors Way, Ann Arbor, Michigan 48108.

\section{THE MODEL}

We consider a uniform, neutral, plasma subject to a uniform external magnetic field, $\mathbf{B}_{0}=\hat{z} B_{0}$. On this equilibrium, a small signal electric field of the form

$$
\mathbf{E}_{\mathrm{l}}=\mathbf{E}_{10} \exp (i \omega t-i \mathbf{k} \cdot \mathbf{x}),
$$

is imposed. From Faraday's law, the accompanying magnetic field perturbation is

$$
\begin{aligned}
& \mathbf{B}_{1}=\mathbf{B}_{10} \exp (i \omega t-i \mathbf{k} \cdot \mathbf{x}), \\
& \mathbf{B}_{10}=\frac{\mathbf{k} \times \mathbf{E}_{10}}{\omega} .
\end{aligned}
$$

For the Alfven wave and the magnetosonic wave, we may focus mainly on the small signal current carried by the singly charged ionic component. For simplicity, we assume that, in equilibrium, the ions have a monoenergetic velocity $v_{\perp}$ perpendicular to the magnetic field, and zero velocity along the field line. Let $\mathbf{x}_{0}=\left(x_{0}, y_{0}, z_{0}\right)$ be the unperturbed position of an ion fluid element. Then

$$
\begin{aligned}
& x_{0}=x_{g}-\frac{v_{\perp}}{\Omega} \sin (\Omega t+\phi), \\
& y_{0}=y_{g}+\frac{v_{\perp}}{\Omega} \cos (\Omega t+\phi), \\
& z_{0}=p=\text { const, }
\end{aligned}
$$

where $\Omega=e B_{0} / m$ is the ion cyclotron frequency and the constants $p, \phi, x_{g}$, and $y_{g}$ are used to label the fluid element. In the presence of perturbation, this element suffers a displacement $x_{1}$ from its equilibrium position. Hence, its instantaneous position is

$$
\mathbf{x}=\mathbf{x}_{0}+\mathbf{x}_{1}\left(\mathbf{x}_{0}, t\right) .
$$

Hereafter, the subscript 1 is used to denote linearized values and the subscript 0 the unperturbed values. The independent 
variables $\left(\mathbf{x}_{0}, t\right)$ in Eq. (4) are known as the polarization variables. ${ }^{2}$ In these variables, the current density perturbation is given by ${ }^{2}$

$$
\mathbf{J}_{1}\left(\mathbf{x}_{0}, t\right)=\frac{\partial}{\partial t}\left(e n_{0} \mathbf{x}_{1}\right)+\nabla \times\left(e n_{0} \mathbf{x}_{1} \times \mathbf{v}_{0}\right),
$$

where $\mathbf{v}_{0}$ is the unperturbed velocity and $n_{0}$ is the density attached to the fluid element. Equation (5) is valid for a general equilibrium density and velocity profile. For the present study, $\mathbf{v}_{0}=\left(\dot{x}_{0}, \dot{y}_{0}, \dot{z}_{0}\right)$ is obtained from (3) and $n_{0}$ is a constant. For perturbation quantities given in the form of Eqs. (1) and (2), Eq. (5) becomes

$$
\mathbf{J}_{1}=e n_{0}\left[i \omega \mathbf{x}_{1}-i \mathbf{k} \times\left(\mathbf{x}_{1} \times \mathbf{v}_{0}\right)\right] \text {. }
$$

Since we are only dealing with small perturbations, we rewrite Eq. (1) as

$$
\mathbf{E}_{1}=\hat{e} E_{10} e^{i \omega t-i \mathbf{k} \cdot \mathbf{x}} \approx \hat{e} E_{10} e^{i \omega t-i \mathbf{k} \cdot \mathbf{x}_{0}},
$$

where $\hat{e}$ is a complex vector of unit magnitude and $E_{10}$ is the (arbitrary) small signal complex amplitude. The Maxwell equations, combined as,

$$
\mathbf{k} \times\left(\mathbf{k} \times \mathbf{E}_{1}\right)+\frac{\omega^{2}}{c^{2}} \mathbf{E}_{1}=i \omega \mu_{0} \mathbf{J}_{1},
$$

yield

$$
\frac{\omega^{2}}{c^{2}}-(\mathbf{k} \times \hat{e}) \cdot\left(\mathbf{k} \times \hat{e}^{*}\right)=\frac{i \omega \mu_{0} \mathbf{J}_{1} \cdot \hat{e}^{*}}{E_{10}} e^{-i \omega t+i \mathbf{k} \cdot \mathbf{x}_{0}} .
$$

Equation (9a) is obtained by substituting Eq. (7) into Eq. (8) and then dot multiplying the result by $\hat{e}^{*}$, where the asterisk denotes the complex conjugate. Since $\mathbf{J}_{1}$ is linearly proportional to $E_{10}$, Eq. (9a) is the dispersion relation, after averaging its right-hand member over a Larmor period. Equation (9a) is a statement of energy conservation: the electromagnetic mode, represented by the left-hand side, is powered by the work done of the radio frequency (RF) current $\mathbf{J}_{1}$ onto the RF electric field $E_{10}$, the latter is represented by the right-hand side of Eq. (9a). Alternatively, Eq. (6) yields the appealing relationship,

$$
\mathbf{J}_{1} \cdot \mathbf{E}_{1}^{*}=e n_{0} i \omega \mathbf{x}_{1} \cdot\left(\mathbf{E}_{1}^{*}+\mathbf{v}_{0} \times \mathbf{B}_{1}^{*}\right),
$$

where use has been made of Eq. (2). The right-hand side of Eq. (9b) is simply the work done per unit volume on the fluid displacement $\mathbf{x}_{1}$ by the small signal electromagnetic force, expressed in complex notation.

Since we are considering a uniform medium, we set $\mathbf{x}_{g}=0$ hereafter.

\section{ALFVÉN WAVE}

We first consider the Alfvén wave, which propagates parallel to the external magnetic field $\hat{z} B_{0}$. We postulate that $\mathbf{E}_{1}$ will be mainly polarized in the $x$ direction. ${ }^{3}$ (This assumption, as well as the role played by the electrons that has been implicitly assumed, is discussed more fully in the last paragraph of this section.) Then $\mathbf{B}_{1}$ is in the $y$ direction, and we have [Fig. 1(a)]

$\mathbf{k}=\hat{z} k_{z}, \quad \hat{e}=\hat{e}^{*}=\hat{x}, \quad \mathbf{B}_{1}=\hat{y} \frac{k_{z} E_{10}}{\omega} e^{i \omega t-i k_{z}^{2}}$. (a) Alfven Wave

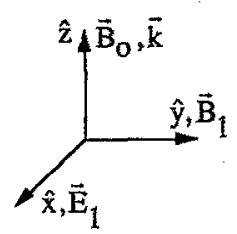

(b) Magnetosonic Wave

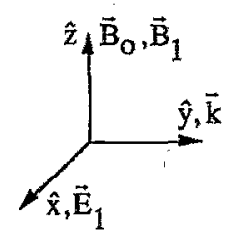

FIG. 1. The propagation vector $\mathbf{k}$, and the electromagnetic fields $\mathbf{E}_{1}$ and $\mathbf{B}_{1}$, for (a) the Alfvén mode and (b) the magnetosonic mode.

If we use the equations in (10) in Eq. (9a), the dispersion relation becomes

$$
\frac{\omega^{2}}{c^{2}}-k_{z}^{2}=\frac{i \omega \mu_{0} J_{1} \cdot \hat{x}}{E_{10}} e^{-i \omega t+i k_{z} z_{0}(t)}
$$

To calculate $J_{1}$, we start from the linearized Lorentz equations,

$$
\begin{aligned}
& \ddot{x}_{1}=-\Omega \dot{y}_{1}+\frac{e}{m} E_{10} e^{i \omega t-i k_{z} p}, \\
& \ddot{y}_{1}=\Omega \dot{x}_{1}, \\
& \ddot{z}_{1}=\frac{e}{m} \dot{x}_{0}\left(\frac{k_{2} E_{10}}{\omega}\right) e^{i \omega t-i k_{2} p} .
\end{aligned}
$$

Equations $(12 a)-(12 c)$ can be solved to yield

$$
\begin{aligned}
x_{1}= & \frac{(e / m) E_{10} e^{i \omega t-i k_{z} p}}{-\omega^{2}+\Omega^{2}}, \\
z_{1}= & -\frac{e}{m}\left(\frac{k_{z} E_{10}}{\omega}\right)\left(\frac{v_{\perp}}{2}\right)\left(\frac{e^{i(\Omega t+\phi)+i \omega t-i k_{z} p}}{-(\omega+\Omega)^{2}}\right. \\
& \left.+\frac{e^{-i(\Omega t+\phi)+i \omega t-i k_{z} p}}{-(\omega-\Omega)^{2}}\right),
\end{aligned}
$$

which may be used in (6) to obtain $\mathbf{J}_{1}$. The dispersion relation (11) then yields, upon averaging over the cyclotron period,

$$
\begin{aligned}
\frac{\omega^{2}}{c^{2}}-k_{z}^{2}= & \frac{\omega_{p}^{2}}{c^{2}}\left[-\frac{\omega^{2}}{\Omega^{2}-\omega^{2}}+\left(\frac{k_{z} v_{\perp}}{2}\right)^{2}\left(\frac{1}{(\omega+\Omega)^{2}}\right.\right. \\
& \left.\left.+\frac{1}{(\omega-\Omega)^{2}}\right)\right],
\end{aligned}
$$

where $\omega_{p}=\sqrt{e^{2} n_{0} / m \epsilon_{0}}$ is the ion plasma frequency. We show in Appendix A that the dispersion relation (14) can be obtained from the Vlasov approach. Thus, this dispersion relation retains all finite Larmor radius effects according to this model [Fig. 1(a)]. It reduces to the well-known results when $v_{\perp} \rightarrow 0$ [cf. Eq. (7.211) on p. 162 of Ref. 3]. As it stands, Eq. (14) yields unstable modes when $\omega_{p}$ and $v_{\perp}$ are sufficiently large.

A few words on the polarization and on the role played by the electrons are in order. Once the propagation vector, $\mathbf{k}$, is specified, the small signal electric field, $\mathbf{E}_{1}$, can no longer be specified independently. It is well known ${ }^{4}$ that the frequency and the associated electromagnetic field, $\mathbf{E}_{1}$, need to 
be determined, respectively, as the eigenvalue and the eigenvector obtained from the conductivity tensor corresponding to the (prespecified) wave vector $k$. In the case of the lowfrequency Alfvern wave, $\mathbf{k} \| \mathbf{B}_{0}$, the small signal current $\mathbf{J}_{1}$ that drives the electric field [Eq. (9a)] originates mainly from the polarization drift of the ionic component. This comes about because (a) the polarization drift of the electronic component is smaller by the mass ratio between an electron and an ion, and (b) the $\mathbf{E}_{1} \times \mathbf{B}_{0}$ drift, which is the same for both electrons and ions, does not contribute to a net current. ${ }^{3}$ Thus, we postulate that the RF electric field is polarized in the $x$ direction and consider only the ionic perturbation current in that direction [Eq. (9a)], as is done in this paper for both the Alfvén wave and the magnetosonic waves. ${ }^{5}$

\section{FAST MAGNETOSONIC WAVE}

The fast magnetosonic wave propagates perpendicular to the external magnetic field, say, in the $y$ direction. Again, we postulate that the electric field is polarized in the $x$ direction. ${ }^{3}$ Thus, we have the following [Fig. 1(b)]:

$$
\begin{aligned}
& \mathbf{k}=k_{y} \hat{y}, \quad \hat{e}=\hat{e}^{*}=\hat{x} \\
& \mathbf{B}_{1}=-\hat{z}\left(\frac{k_{y} E_{10}}{\omega}\right) e^{i \omega t-i k_{y} y_{0}} .
\end{aligned}
$$

Equation (9a) becomes

$$
\frac{\omega^{2}}{c^{2}}-k_{y}^{2}=\frac{i \omega \mu_{0} \mathrm{~J}_{1} \cdot \hat{x}}{E_{10}} e^{-i \omega t+i b \cos (\Omega t+\phi)},
$$

where $b=k_{y} v_{\perp} / \Omega$.

The Lorentz force law is linearized to yield

$$
\begin{aligned}
& \ddot{x}_{1}=\frac{e}{m}\left[E_{1 x}+\dot{y}_{0}\left(\frac{-k_{y} E_{1 x}}{\omega}\right)\right]-\Omega \dot{y}_{1}, \\
& \ddot{y}_{1}=\Omega \dot{x}_{1}-\frac{e}{m} \dot{x}_{0} B_{1 z}, \\
& \ddot{z}_{1}=0,
\end{aligned}
$$

where $E_{1 x}=E_{10} e^{i \omega t-i b \cos (\Omega t+\phi)}, B_{1 z}=-k_{y} E_{1 x} / \omega$, and $\dot{x}_{0}$ and $\dot{y}_{0}$ are equilibrium velocities derived from Eq. (3). To solve these differential equations we write the solutions in the form

$$
\begin{aligned}
& x_{1}=\frac{e E_{10}}{m \Omega^{2}} \sum_{n=-\infty}^{\infty} \xi_{n} e^{i \omega t-i n(\Omega t+\phi)}, \\
& y_{1}=\frac{e E_{10}}{m \Omega^{2}} \sum_{n=-\infty}^{\infty} \eta_{n} e^{i \omega t-i n(\Omega t+\phi)} .
\end{aligned}
$$

Substitution of Eqs. (18a) and (18b) into Eqs. (17a) and (17b) yields

$$
\begin{aligned}
& \xi_{n}=\frac{J_{n}(b)(\bar{\omega}-n)^{2}+b J_{n}^{\prime}(b)}{\bar{\omega}(\bar{\omega}-n)\left[-(\bar{\omega}-n)^{2}+1\right]} e^{-i n \pi / 2}, \\
& \eta_{n}=\frac{(\bar{\omega}-n) \xi_{n}}{i}+\frac{1}{i \bar{\omega}} J_{n}(b) e^{-i n \pi / 2},
\end{aligned}
$$

where $\bar{\omega}=\omega / \Omega$ and a prime $\left({ }^{\prime}\right)$ denotes the derivative with respect to the argument. We have used the Bessel function identities,

$$
\begin{aligned}
& e^{i b \cos \phi}=\sum_{n=-\infty}^{\infty} J_{n}(b) e^{ \pm i n \phi+i n \pi / 2}, \\
& e^{-i b \cos \phi}=\sum_{n=-\infty}^{\infty} J_{n}(b) e^{ \pm i n \phi-i n \pi / 2},
\end{aligned}
$$

in which either the + or - sign may be used in $( \pm)$ in the right-hand members. We insert (18a) and (18b) into (6), and take the $x$ component. Equation (16) then gives, upon averaging the right-hand side over a cyclotron period, ${ }^{1}$

$$
\begin{aligned}
\frac{\omega^{2}}{c^{2}} k_{y}^{2}= & \frac{\omega_{p}^{2}}{c^{2}} \bar{\omega} \sum_{n=-\infty}^{\infty}\left(-\tilde{\omega} \xi_{n}+\frac{b}{2}\left[i\left(\xi_{n+1}-\xi_{n-1}\right)\right.\right. \\
& \left.\left.+\eta_{n+1}+\eta_{n-1}\right]\right) J_{n}(b) e^{i n \pi / 2}
\end{aligned}
$$

which may be simplified to read as

$$
\frac{\omega^{2}}{c^{2}}-k_{y}^{2}=\frac{\omega_{p}^{2}}{c^{2}}\left(1+\sum_{n} \frac{\left[J_{n}(b)+b J_{n}^{t}(b)\right]^{2}}{(\bar{\omega}-n)^{2}-1}\right)
$$

The following Bessel identities have been used:

$$
\begin{aligned}
& \sum_{n} J_{n}(b)\left[J_{n-1}^{\cdots}(b)-J_{n+1}^{\mathrm{s}}(b)\right]=0, \cdots \\
& \sum_{n} J_{n}(b) J_{n}^{\prime}(b)=0, \quad J_{-n}(b)=(-1)^{n} J_{n}(b), \\
& \sum_{n} J_{n}^{2}(b)=1 .
\end{aligned}
$$

In Eqs. (22) and (23), the summation index $n$ runs from $-\infty$ to $\infty$. In Appendix B, we shall recover the same dispersion relation (22) using the Vlasov approach. This dispersion relation yields unstable mode solutions when $\omega_{p}$ and $v_{\perp}$ are sufficiently large. Equation (22) reduces to the well-known result $^{3}$ as $v_{\perp} \rightarrow 0$, i.e., as $b \rightarrow 0$.

\section{DISCUSSIONS}

The reconstruction of the major electrostatic modes in I and the electromagnetic modes that is given here still makes one wonder whether the fluid approach is capable of offering an equivalent formulation of all kinetic effects. While we have so far only explicitly demonstrated the equivalence for a uniform plasma, ${ }^{5}$ we believe that such a linearized fluid theory may be extended to a nonuniform plasma, as Eq. (5) is valid for a nonuniform medium. ${ }^{2}$

In a "weakly" nonuniform plasma, the eikonal approximation is essentially one in which the equilibrium quantities are regarded as a local constant. Thus, the expression for $\mathbf{J}_{1}$, 
Eq. (6), gives the current response in a weakly nonuniform plasma under the eikonal approximation. It accounts for all kinetic effects under such an approximation.

The a local theory clearly needs modification when there is a sharp boundary, or when the local wavelength is comparable to the inhomogeneous scale length. However, in the latter case, considerable insight may still be gained, as the asymptotic theories are known to yield reliable answers even when the (small or large) expansion parameter in such theories turns out to be of order unity.

There are subtleties that require some discussions. In any treatment of electromagnetic modes, the propagation vector $\mathbf{k}$ is first specified. The polarization in $\mathbf{E}_{1}$ needs to be calculated and cannot be independently assigned. ${ }^{3,4}$ In our treatment of both kinetic Alfvén waves and magnetosonic waves, we did specify a priori the polarization in $\mathbf{E}_{1}$ in addition to $\mathbf{k}$. In spite of these potential inconsistencies, the finite Larmor radius effects from both the fluid approach and from the Vlasov approach turn out to be identical. This is even more remarkable in the case of the magnetosonic waves. In its fluid description, the perturbed displacement $\mathbf{x}_{1}$, and therefore the perturbed current density $\mathbf{J}_{1}$, depends on the perturbed magnetic field $\mathbf{B}_{1}=\mathbf{k} \times \mathbf{E}_{1} / \omega$, which admittedly may be inherently inconsistent, since the electric field $\mathbf{E}_{1}$ has been assigned a priori. On the other hand, in the Vlasov approach, the perturbation current density $\mathbf{J}_{1}$ does not even depend on the perturbed magnetic field [cf. Eq. (B2) and the last paragraph of Appendix B]. Identical dispersion relations are obtained, however.

Questions may also be raised on the justification of replacing the curl operator $(\nabla \times)$ in Eq. (5) simply by $(-i \mathbf{k} \times)$ in Eq. (6). This is not an easy question to answer, even though this simple replacement apparently yields the correct answer for a uniform medium. ${ }^{5}$ Care is to be exercised in an analysis involving an equilibrium flow with velocity shear. Thus, while we have demonstrated that the fluid theory is able to formulate all kinetic effects without explicitly solving the linearized Vlasov equation, it remains to be seen to what extent the present fluid theory of kinetic modes can profitably be applied to a spatially nonuniform plasma, or to a nonlinear theory under the eikonal approximation.

\section{ACKNOWLEDGMENTS}

This work is supported in part by the Naval Research Laboratory, and by the Office of Naval Research.

\section{APPENDIX A: ALFVÉN WAVE-VLASOV APPROACH}

In this appendix, we obtain the dispersion relation for the kinetic Alfvén waves from the Vlasov formulation. For the polarization given in Fig. 1(a), we need to calculate the current response $J_{1 x}$ in Eq. (8):

$$
\left(\frac{\omega^{2}}{c^{2}}-k_{z}^{2}\right) E_{1 x}=i \omega \mu_{0} J_{1 x},
$$

where

$$
\begin{aligned}
J_{1 x} & =e n_{0} \int d^{3} v\left(v_{x} f_{1}\right) \\
& =e n_{0} \int_{0}^{\infty} d v_{\perp} \int_{0}^{2 \pi} v_{\perp} d \phi \int_{-\infty}^{\infty} d v_{z}\left(v_{\perp} \cos \phi\right) f_{1} .
\end{aligned}
$$

The perturbed distribution function $f_{1}$ in Eq. (A2) is determined from an integration over the unperiurbed orbits: ${ }^{7}$

$$
f_{1}=\int_{-\infty}^{t} d t^{\prime}\left(-\frac{e}{m}\right)\left[\mathbf{E}_{1}^{\prime}+\mathbf{v}^{\prime} \times \mathbf{B}_{1}^{\prime}\right] \cdot \frac{\partial f_{0}}{\partial \mathbf{v}^{\prime}},
$$

where $f_{0}=f_{0}\left(v_{1}, v_{z}\right)$ is the equilibrium distribution function and the primes ( $\left.{ }^{\prime}\right)$ designate quantities evaluated along the unperturbed orbits. The equations for the unperturbed orbits are

$$
\begin{aligned}
& v_{x}\left(t^{\prime}\right)=v_{\perp} \cos \left[\Omega\left(t^{\prime}-t\right)+\phi\right], \\
& v_{y}\left(t^{\prime}\right)=v_{\perp} \sin \left[\Omega\left(t^{\prime}-t\right)+\phi\right], \\
& v_{z}\left(t^{\prime}\right)=v_{z}, \\
& x\left(t^{\prime}\right)=\frac{v_{\perp}}{\Omega} \sin \left[\Omega\left(t^{\prime}-t\right)+\phi\right]-\frac{v_{\perp}}{\Omega} \sin \phi+x, \\
& y\left(t^{\prime}\right)=-\frac{v_{\perp}}{\Omega} \cos \left[\Omega\left(t^{\prime}-t\right)+\phi\right]+\frac{v_{\perp}}{\Omega} \cos \phi+y, \\
& z\left(t^{\prime}\right)=v_{z}\left(t^{\prime}-t\right)+z,
\end{aligned}
$$

so that at $t^{\prime}=t, \quad v_{x}\left(t^{\prime}\right)=v_{x}=v_{\perp} \cos \phi$, $v_{y}\left(t^{\prime}\right)=v_{y}=v_{\perp} \sin \phi, v_{z}\left(t^{\prime}\right)=v_{z}, x\left(t^{\prime}\right)=x, y\left(t^{\prime}\right)=y$, and $z\left(t^{\prime}\right)=z$. Then using these expressions and Eqs. (1) and (2) of the main text into Eq. (A3), we obtain

$$
\begin{aligned}
f_{1}= & -\frac{e E_{10}}{2 m}\left[\left(1-\frac{v_{z} k_{z}}{\omega}\right) \frac{\partial f_{0}}{\partial v_{\perp}}+\frac{v_{\perp} k_{z}}{\omega} \frac{\partial f_{0}}{\partial v_{z}}\right] \\
& \times\left(\frac{e^{i \phi}}{i\left(\omega-\Omega-k_{z} v_{z}\right)}+\frac{e^{-i \phi}}{i\left(\omega+\Omega-k_{z} v_{z}\right)}\right) .
\end{aligned}
$$

In this paper, we consider only a monoenergetic plasma with the equilibrium distribution function:

$$
f_{0}(\mathbf{v})=\frac{1}{2 \pi v_{\perp}} \delta\left(v_{\perp}-v_{\perp 0}\right) \delta\left(v_{z}\right),
$$

where $\delta$ is the Dirac delta function. Upon substituting Eq. (A6) into (A5), and the resultant expression into Eq. (A2), we obtain from Eq. (A 1):

$$
\begin{aligned}
\frac{\omega^{2}}{c^{2}}-k_{z}^{2}= & \frac{\omega_{p}^{2}}{c^{2}}\left[-\frac{\omega^{2}}{\Omega^{2}-\omega^{2}}+\left(\frac{v_{\perp} k_{z}}{2}\right)^{2}\left(\frac{1}{(\omega+\Omega)^{2}}\right.\right. \\
& \left.\left.+\frac{1}{(\omega-\Omega)^{2}}\right)\right],
\end{aligned}
$$

which is identical to Eq. (14), the dispersion relation constructed in Sec. III using the fluid approach. 


\section{APPENDIX B: FAST MAGNETOSONIC WAVE- VLASOV APPROACH}

For the magnetosonic wave [Fig. (1b)], the dispersion relation ( 8 ) becomes

$$
\left(\frac{\omega^{2}}{c^{2}}-k_{y}^{2}\right) E_{1 x}=i \omega \mu_{0} J_{1 x}
$$

and $J_{1 x}$ is given by Eq. (A2).

Again, we determine the perturbed distribution function by integrating over unperturbed orbits. For the magnetosonic wave, with electromagnetic fields as specified in Fig. 1(b), Eq. (A.3) may be simplified to read as

$$
f_{1}=\int_{-\infty}^{t} d t^{\prime}\left(-\frac{e}{m}\right)\left(\mathbf{E}_{1}^{\prime} \cdot \frac{\partial f_{0}}{\partial \mathbf{v}^{\prime}}\right) \text {. }
$$

If we let $\bar{\omega} \equiv \omega / \Omega$ and $b \equiv k_{y} v_{\perp} / \Omega$ as in Sec. IV, and use Eq. (7) and the unperturbed orbit equations. (A4), we obtain, from Eq. (B2),

$$
\begin{aligned}
f_{1}= & -\frac{e E_{10}}{m \Omega}\left(\frac{\partial f_{0}}{\partial v_{1}}\right) e^{i \omega t-i k_{y} y-i b \cos \phi} \\
& \times \sum_{n=-\infty}^{\infty} J_{n}(b) \frac{e^{i n \pi / 2}}{2}\left(\frac{e^{-i(n-1) \phi}}{i(\bar{\omega}-n+1)}\right. \\
& \left.+\frac{e^{-i(n+1) \phi}}{i(\bar{\omega}-n-1)}\right)
\end{aligned}
$$

where we have used the Bessel function identities (20). We next use the unperturbed distribution function (A6) in Eq. (B3), and substitute the resultant expression in Eq. (A2). The dispersion relation $(\mathrm{B} 1)$ then reads as

$$
\begin{aligned}
\frac{\omega^{2}}{c^{2}}-k_{y}^{2}= & \left(\frac{\omega}{\Omega}\right) \frac{\omega_{p}^{2}}{c^{2}}\left(\frac{1}{b}\right) \\
& \times \sum_{m=-\infty}^{\infty} \frac{(d / d b)\left\{\left[b(d / d b) J_{m}(b)\right]^{2}\right\}}{\bar{\omega}-m},
\end{aligned}
$$

where we have used the following Bessel function relations:

$$
\begin{aligned}
& J_{m}^{\prime \prime}(b)=-\frac{1}{b} J_{m}^{\prime}(b)-\left(1-\frac{m^{2}}{b^{2}}\right) J_{m}(b) \\
& J_{m-1}(b)-J_{m+1}(b)=2 J_{m}^{\prime}(b) \\
& J_{m-1}(b)+J_{m+1}(b)=\frac{2 m}{b} J_{m}(b)
\end{aligned}
$$

Equation (B4) can be shown to be equivalent to Eq. (22) of Sec. IV.

It is interesting to note that even though the fluid approach and the Vlasov approach produce equivalent dispersion relations, the $\mathbf{v} \times \mathbf{B}_{1}$ term is absolutely necessary in the fluid approach [cf. Eqs. (9a) and (9b)], but does not contribute to the Vlasov theory of the magnetosonic waves [cf. Eq. (B2)].

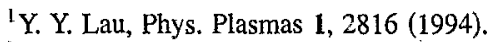

${ }^{2}$ D. L. Bobrotf, IRE Trans. Electron. Devices ED-6, 68 (1959).

${ }^{3}$ See, e.g., D. R. Nicholson, Introduction to Plasma Theory (Wiley, New York, 1983), Chap. 7.

${ }^{4}$ See, e.g., T. H. Stix, The Theory of Plasma Waves (McGraw-Hill, New York, 1962), Chap. 8.

${ }^{5}$ We have also used the fluid approach given in this paper to reconstruct the dispersion relation for the Weibel instability [E. S. Weibel, Phys. Rev. Lett. 2,83 (1959)]. In this case, the polarization of the small signal electric field and the role played by the electrons are vastly different from the kinetic Alfvén mode, even though both modes are electromagnetic and are characterized by $\mathbf{k} / / \mathbf{B}_{0}$. The Weibel instability is a high-frequency kinetic mode, in which the ions may be taken as infinitely massive. For an electron equilibrium distribution function given by Eq. (A6) of Appendix A, the small signal electric field, $\mathbf{E}_{1}$, is no longer linearly polarized. By assuming a general polarization orthogonal to the external magnetic field, we solved for the fiuid displacement $\mathbf{x}_{1}$ in response to $\mathbf{E}_{1}$ [and to $\mathbf{B}_{1}$ by virtue of Eq. (2)]. We next used Eq. (6) to recalculate the small signal current density, $\mathbf{J}_{1}$. The eigenvector $\mathbf{E}_{1}$ constructed out of $\mathbf{E q}$. (8) for the electromagnetic modes then turns out to be circularly polarized, and the dispersion relation obtained from this fluid reconstruction is identical to Eq. $\left(8^{\prime}\right)$ of K. R. Chu and J. L. Hirshfield [Phys. Fluids 21, 461 (1978)]. 